\title{
Die Vielfalt semantischer Komplexbildungsweisen
}

\author{
Von STEFAN TOLKSDORF (Berlin)
}

Das Problem der Einheit der Proposition stellt nicht nur eines der Gründungsprobleme der analytischen Philosophie dar, sondern ist darüber hinaus auch Gegenstand gegenwärtiger philosophischer Auseinandersetzungen. Dieses neuerliche Aufflammen einer bekannten Thematik, beispielsweise in den Arbeiten von Martha Gibson, Donald Davidson oder Richard Gaskin $^{1}$, ist ein deutliches Anzeichen dafür, dass bisher keine zufriedenstellende Antwort gefunden wurde. Die Brisanz der Frage, aber auch die Komplexität möglicher Antwortstrategien hängen nicht zuletzt mit der Tatsache zusammen, dass die ,Einheit der Proposition“ auf vielen Gebieten der Philosophie diskutiert wird. In diesem Sinne bemerkt Davidson:

„The philosophy of language lacks its most important chapter without such a theory; the philosophy of mind is missing a crucial first step if it cannot describe the nature of judgement; and it is woeful if metaphysics cannot say how a substance is related to its attributes." 2

Hinter der Ausgangsfrage, so legt dieses Zitat nahe, verbirgt sich, streng genommen, eine dreifache Herausforderung: die Einheit des Satzes, die Einheit des Urteilen und Denkens und die Einheit des Sachverhalts. Für den Fortgang der Untersuchung sei folgende Entscheidung getroffen: Ich werde mich in erster Linie mit der sprachphilosophischen Fragestellung nach der Einheit des Satzes auseinandersetzen. Hinsichtlich des inneren Zusammenhangs dieser drei Probleme plädiere ich im Sinne der Sprachphilosophie dafür, dass wir es hier nicht mit drei isoliert voneinander verständlichen, eigenständigen Problemen zu tun haben, da die Einheit des Satzes eine explanatorische Priorität der Art besitzt, dass aus dieser Schlussfolgerungen über die Komplexität des Urteilensaktes und der Sachverhalte gezogen werden können. In diesem Sinne geht die Sprachphilosophie der Metaphysik und der Philosophie des Geistes voraus.

Bezogen auf den Fokus meiner Abhandlung stimme ich Davidson zu, dass wir keine Sprachphilosophie betreiben können, ohne etwas zur semantischen Komplexität von Sätzen zu sagen. Verstehen wir die Art der Komplexität der zentralen sprachlichen Züge nicht, dann bleibt unklar, was einen Satz als Satz auszeichnet. Damit aber bliebe das sprachphilosophische Explanandum unverstanden.

1 Vgl. M. Gibson, From Naming to Saying, Weinheim 2004; D. Davidson, Truth and Predication, Cambridge/Mass. 2005; R. Gaskin, The Unity of Proposition, Oxford 2009.

2 D. Davidson, Truth and Predication, a. a. O., 77. 


\section{Das Problem der Einheit des Satzes: Komplexität, Sinn und Bedeutung}

Nachdem ein erster Grenzstein gesetzt und auf die Zentralität der Fragestellung hingewiesen wurde, wende ich mich nun der Ausgangsfrage zu. Wir sollten zuerst verstehen, worin das Problem der Einheit des Satzes eigentlich besteht. Von besonderer Bedeutung ist dabei die Beobachtung, dass ein Satz mehr ist als die Summe seiner Teile, das heißt mehr ist als die Zusammenstellung von Wörtern. Das bedeutet, dass sich Sätze von Namens- beziehungsweise (allgemeiner) Wortlisten darin unterscheiden, dass erstere eine inhaltliche Struktur besitzen beziehungsweise über eine Art semantischer Komplexität verfügen. Wer den Satz äußert, Der Tisch ist braun", der vollzieht nicht zwei beziehungsweise drei isolierte Äußerungen der Art ,der Tisch, ... ist braun“ oder ,der Tisch, Bräune, Eigenschafts-Exemplifikation', sondern sagt, dass der Tisch braun ist. Die Elemente des Satzes treten zu einer Bedeutungseinheit zusammen, deren Strukturiertheit es verbietet, die semantischen Teile willkürlich (gleichwohl syntaktisch erlaubt) zu verschieben. Bezogen auf die Bedeutungseinheit des Satzes ist die Bemerkung Wittgensteins zu verstehen, dass einzig das Äußern eines Satzes ein Zug im Sprachspiel darstellt. Mit anderen Worten: Sätze sind die kleinsten Einheiten sprachlichen Handelns.

Wir können also festhalten, dass die Frage nach der Einheit des Satzes eine Erklärung dafür verlangt, wie die Rede von der komplexen Bedeutungseinheit des Satzes, gedeutet als Komplexität der sprachlichen Handlung, zu verstehen ist. Darauf komme ich gleich zurück.

Dabei ist es wichtig, gleich zu Beginn auf eine Adäquatheitsbedingung einer jeden solchen Erklärung aufmerksam zu machen. Diese ergibt sich daraus, dass der Verweis auf Komplexität alleine nicht ausreicht, um Sätze von Namenslisten zu unterscheiden. Letztere sind nicht zuletzt deshalb komplex, weil eine Liste aus verschiedenen Einträgen besteht, eine bestimmte Reihenfolge aufweist. Selbst wenn wir sagen, dass die Einträge der Liste willkürlich verändert werden können, was bei einem Satz nicht ohne weiteres möglich ist, haben wir damit noch keine Erklärung der Differenz gegeben. Worauf damit aber hingewiesen wird, ist die Tatsache, dass Arten von Komplexbildungsweisen unterschieden werden müssen. Die Komplexitätsstruktur der Namensliste entsteht durch das aggregathafte Aneinanderstellen einzelner Namen, wobei jeder Eintrag auf eigenen Füßen steht (etwas bezeichnet). Das heißt, dass die Teile ihren Beitrag unabhängig von der Existenz anderer Teile leisten. Genau das aber trifft auf semantische Komplexität nicht zu. In dem Satz ,Der Tisch ist braun“ ist die Rede von zwei oder mehr Teilen nicht so zu verstehen, dass zwei isolierte, für sich allein verständliche Züge aneinander gebunden werden, die im Prinzip auch getrennt auftreten könnten. Darüber hinaus ist das sprachlich komplexe Zeichen ,Der Tisch ist braun“ in ,Ich glaube nicht, dass der Tisch braun ist" enthalten, nicht aber der damit verbundene Zug im Sprachspiel. Für semantische Komplexbildungsweisen ist es durchaus üblich, dass Zeichen auf der Ebene des Satzes eine Einheit bilden, ohne damit implizieren zu müssen, die Teilhandlungen des Satzes würden einzig durch das Nacheinander der Vollzüge zu einem Handlungskomplex. Aus diesen Gründen ist beispielsweise der Ansatz Wittgensteins im Tractatus zu verwerfen:

„4.22: Der Elementarsatz besteht aus Namen. Er ist ein Zusammenhang, eine Verkettung, von Namen.

4.221: Es fragt sich hier, wie kommt der Satzverband zustande. “3

3 Vgl. L. Wittgenstein, Tractatus logico-philosophicus, 12. Aufl., Frankfurt/M. 1999. 
Wittgenstein fragt hier zu Recht nach der Verkettung, nach dem Satzverband, jedoch scheint die in Aussicht gestellte Lösung, Namen zu verbinden, für die geforderte Art der Komplexität kaum geeignet. Ganz im Sinne unserer Unterscheidung aggregathafter von semantischer Komplexität bemerkt Frege in diesem Zusammenhang:

„Aus dem Namen der Zahl 2 und dem Namen für den Begriff der Primzahl erhält man nicht den Satz ,2 ist eine Primzahl', sondern die Namensliste ,2 der Begriff der Primzahl'."“4

Damit ist natürlich die Einheit des Satzes noch nicht erklärt, lediglich genauer umrissen, was es zu erklären gilt.

Bevor ich fortfahre, müssen weitere Entscheidungen getroffen und Weichenstellungen vorgenommen werden. Gesagt wurde, dass es um die Frage geht, wie die semantische Komplexität von Sätzen verstanden werden kann. Der Bezug auf den Inhalt von Sätzen macht deutlich, dass wir nicht an ,formalen' Gesichtspunkten interessiert sind, wie beispielsweise der akustischen, grafischen oder syntaktischen Einheit eines, Satzes'. Damit bleibt jedoch immer noch offen, ob die Einheit des Satzes primär eine Eigenschaft des Sinns oder aber des Bezugs (der Fregeschen Bedeutung) ist. Diese Offenheit der Frage wurzelt in der Mehrdeutigkeit der Rede von der Bedeutung eines Satzes und spiegelt sich in der Mehrdeutigkeit des Ausdrucks ,Proposition“ wider, welcher zwischen Satzsinn (Gedanke, propositionaler Gehalt) und Satzbezug (Wahrheitswert etc.) schillert. Meine erste Weichenstellung besteht darin, die Einheit des Satzes primär als ein Problem des Sinns aufzufassen. An dieser Stelle mögen einige wenige Bemerkungen genügen, die gegen das alternative, auf den Bezug ausgerichtete Vorgehen sprechen. Erstens ist mit einer solchen Ausrichtung die Annahme verbunden, wir könnten ähnlich der Kategorie der Gegenstandsnamen auch von prädikativen Ausdrücken und ganzen Sätzen von einem Bezugsobjekt sprechen. Bekanntermaßen sind die entsprechenden Vorschläge, erinnert sei an Extensionen, Begriffe und Wahrheitswerte, heftiger Kritik ausgesetzt. Zweitens führt uns die Rede von der inhaltlichen Struktur des Satzes dazu, die Teile eines solchen so zu deuten, dass sie Elemente eines geordneten Geflechts sind. Was aber soll es vor diesem Hintergrund bedeuten zu sagen, dass der Satzbezug die Bezüge der semantischen Teile in sich aufnimmt? Folgen wir beispielsweise um des Arguments willen Frege, dann müssen wir etwa sagen: Der Bezug des Ausdrucks , die Hauptstadt von Deutschland' (das ist: die Stadt Berlin) geht in den Wahrheitswert des Satzes ,Berlin ist die Hauptstadt von Deutschland“ ein. Das aber kann nicht stimmen. Begriffe und Gegenstände sind kein echter Teil der Satzbedeutung. Das gilt auch für Prädikationen im engeren Sinne, d. h. auch für Sätze der Form F(a), nicht nur für Identitätssätze $(\mathrm{a}=\mathrm{b})$. Drittens haben wir bereits festgehalten, dass der Verweis auf die Referenzobjekte der jeweiligen Elemente eines Satzes im Verdacht steht, die Einheit des Satzes nicht erklären zu können. Am Ende einer solchen Auflistung von referentiellen Zuordnungen steht möglicherweise eine Liste von Entitäten ganz unterschiedlicher Art (Einzeldinge und Universalien; Gegenstände und Begriffe etc.), aber eben keine Einheit. Ich stimme Davidson zu, wenn er sagt:

„To say that predicates are functional expressions, and are therefore incomplete or unsaturated, and that what they refer to is similarly full of holes or spaces waiting to be filled in, does not help: entities are entities, whatever we call them. ${ }^{\text {" }}$

4 Vgl. G. Frege, Über Begriff und Gegenstand, in: ders., Kleine Schriften, Darmstadt 1967, 178.

5 D. Davidson, Truth and Predication, a. a. O., 156. 
Wir sind damit also auf die Ebene des Sinns als primäre Ebene der Einheit des Satzes verwiesen. An dieser Stelle ist eine zweite Weichenstellung notwendig. Frege verband den Sinn eines Satzes mit dem durch den Satz ausgedrückten Gedanken, mit dem , beurteilbaren Inhalt . In eine bekannte Terminologie übersetzt, können wir sagen: Der Sinn des Satzes besteht in seinen Wahrheitsbedingungen, jenen Bedingungen also, die erfüllt sind, wenn der Satz wahr ist. Die Bedingungen der Wahrheit konstituieren die Proposition des Satzes. Die Rede vom Sinn der semantischen Teile kann dann so verstanden werden, dass die Art des Beitrags und der Beitrag selbst, die beziehungsweise den das Wort zur Bestimmung der Wahrheitsbedingung des Satzes leistet, den Sinn festlegen. Ein Begriffszeichen leistet der Art nach einen anderen Beitrag als ein Gegenstandsname. Wenn von der semantischen Rolle eines Zeichens im Satzzusammenhang die Rede ist, dann geht es um die so bestimmte Art des Beitrags zur Bestimmung des vom Satz ausgedrückten Gedankens.

Dieser Beschreibung des Sinns will ich nicht widersprechen. Betont werden soll vielmehr, dass verschiedene Interpretationen der Rede vom Gedanken eines Satzes möglich sind. Der frühe Frege hatte ein realistisches Verständnis der Ebene des Sinns im Auge, ging er doch von einem zeitlosen ,Reich der Gedanken' aus. Ich möchte im Fortgang einen anderen Weg einschlagen und den Sinn eines Satzes und die semantische Rolle der Zeichen pragmatisch deuten, das heißt: bezogen auf den Satz als Handlungsvollzug und bezogen auf die Wörter als Gebrauch. Die zweite Weichenstellung lässt sich daher wie folgt zusammenfassen: Die Einheit des Satzes stellt sich auf der Ebene des Sinns als die Einheit einer komplexen sprachlichen Handlung dar. Es gilt zu verstehen, wie der Sinn der Teile einen Beitrag zur Sprechhandlung leistet. Die semantische Rolle der Zeichen im Satz muss sich demnach an ihrem Gebrauch nachweisen lassen. Wie im nächsten Abschnitt gezeigt wird, hat Frege diesbezüglich einen interessanten Vorschlag zu machen.

\section{Freges funktionale Deutung prädikativer Zeichen}

Frege versuchte, die Einheit des Satzes dadurch zu erklären, dass er von einer kategorialen Differenz zwischen Gegenstandszeichen und Begriffszeichen beziehungsweise Gegenständen und Begriffen ausging. Für die Einheitsbildung ist die prädikative Natur des Begriffs verantwortlich. Diese prädikative Natur hat Frege zumeist metaphorisch umschrieben. So ist die Rede davon, dass Begriffe ungesättigt, unvollständig und ergänzungsbedürftig sind. Die eigentümliche und zugleich wesentliche Offenheit des Begriffs soll die Komplexbildung ermöglichen, indem ein gesättigter Gegenstand an die Leerstelle des Begriffs tritt und so letzteren zu einer Einheit ergänzt.

Das Bild von den gesättigten und ungesättigten Elementen lässt sich bei Frege interessanterweise auf drei Ebenen zur Geltung bringen, was jeweils zu einer anderen Interpretation der ,prädikativen Leerstelle' führt. Diese Unbestimmtheit zeigt sich bereits daran, dass Frege nicht immer deutlich macht, ob es ihm um Gegenstandsnamen (beziehungsweise Begriffszeichen) oder Gegenstände (beziehungsweise Begriffe) geht. In den frühen Schriften wird der Unterschied zwischen ,Funktion ‘ und ,Argument' zumeist formal erläutert, das heißt konkret, bezogen auf den Vorgang des Ersetzens und Konstanthaltens von Ausdrücken. Wird ein Ausdruck aus einem Satzverbund entfernt und die dadurch entstandene Leerstelle entsprechend markiert, dann kann leicht nachvollzogen werden, dass ein solches Gesamtzeichen , ungesättigt $^{`}$ ist. Die Rede von vollständigen und unvollständigen Elementen bezieht sich dabei in einem nicht-metaphorischen Sinne auf die grafischen Zeichenvorkommnisse. Die Zuordnung eines Zeichens in den Kategorien ,Funktion' und ,Argument' bleibt dabei eine Sache der 
nicht auf den Inhalt ausgerichteten Ersetzung eines Zeichens - und zwar so, dass jedes Argument auch hätte Funktion sein können und umgekehrt.

Die Standardinterpretation Freges bezieht sich dagegen nicht auf das Zeichen, sondern auf das Bezeichnete. Frege deutet an vielen Stellen an, dass Begriffe für ihn die Bedeutungen der Begriffsausdrücke sind. Unter diesen Bedingungen müsste die Einheit des Satzes erklärt werden, indem auf unvollständige Entitäten verwiesen wird, die durch vollständige Entitäten ergänzt werden. Die Probleme eines solchen Ansatzes wurden bereits erwähnt.

Ich möchte dagegen betonen, dass der späte Frege seine bildhafte Rede von der Ergänzungsbedürftigkeit immer enger am Sinn der Zeichen ausrichtet. Zu nennen sei hier beispielsweise:

„Eigentlich kommt das Ungesättigtsein im Gebiete des Sinns vor und wird von dort auf das Zeichen übertragen."

$\mathrm{Zu}$ dieser Wandlung vom Bezug zum Sinn kommt nun flankierend eine weitere Entwicklung Freges hinzu, die zu einer pragmatischen Deutung des Sinns führt. Der erwähnten Textstelle geht der folgende Satz voraus:

„Als bloßes Ding ist die Gruppe von Buchstaben ,UND“ freilich ebenso wenig ungesättigt als irgendein anderes Ding. Im Hinblick auf seine Gebrauchsweise als Zeichen, das einen Sinn ausdrücken soll, kann man es ungesättigt nennen, indem es hier nur in der Stellung zwischen zwei Sätzen den gemeinten Sinn haben kann. ${ }^{67}$

Dieses Zitat legt den Gedanken nahe, dass der Sinn eines Zeichens in der Weise des Gebrauchs besteht, die wir von diesem machen. Ungesättigt ist das Wort ,und' deshalb, weil wir es einzig so verwenden, dass es zwischen bereits vorhandenen Zeichenketten zur Geltung kommt. Allgemein können wir sagen, dass prädikative Ausdrücke ungesättigt sind, weil ihr Gebrauch immer schon auf andere Zeichen und deren Verwendung angewiesen ist. Schauen wir dazu das Prädikat ,... ist rot $^{\star}$ an. Pragmatisch verstanden macht der sprachliche Handlungsvollzug der Zuschreibung einer Farbe nur dann Sinn, wenn in diesem Vollzug auf etwas verwiesen wird, das rot sein kann oder nicht. Frege deutet diese intrinsische Bezugnahme eines Begriffs auf andere, gesättigte Zeichen mit der Leerstelle des prädikativen Ausdrucks an: ... ist rot. Farbprädikative verstehen heißt also, dass wir wissen müssen, auf welche Art und Weise Ausdrücke dieser semantischen Art auf andere Handlungskompetenzen (das heißt: auf andere Wörter, zum Beispiel Eigennamen etc.) Bezug nehmen, sie komplexbildend modifizieren. Aus der einfachen sprachlichen Handlung eines Rufes wie Frieda!, die im Sinne Freges ,gesättigt‘ genannt werden kann, weil es sich hier um einen basalen sprachlichen Zug handelt, der aus sich heraus verständlich ist (Personen und Gegenstände unserer Handlungswelt werden angesprochen beziehungsweise benannt, mittels sprachlicher Zeichen herausgegriffen und in ein Spiel eingebunden), wird die komplexe Handlung, Frieda ist zu klein‘. Natürlich gibt Frege die Ungesättigtheit des Bezuges nicht auf. Es soll lediglich auf eine Unentschlossenheit Freges hingewiesen werden. Diese eröffnet die Option, die metaphorischen Umschreibungen primär auf den Sinn zu beziehen.

An diesem Beispiel können wir zwei bereits erwähnte Punkte verdeutlichen. Erstens besteht die oben semantisch genannte Komplexitätsform gerade darin, dass zwar das Zeichen ,Frieda' Element des Satzes ist und als solches darauf verweist, dass mit diesem selbst unter

6 G. Frege, Gedankengefüge, in: ders., Kleine Schriften, Darmstadt 1967, 381.

7 Ebd. 
anderen Bedingungen ein bestimmter Zug im Sprachspiel verbunden ist, dass aber eben nicht dieser Zug im komplexen Satz vollzogen wird. Wer sagt ,Dieser Tisch ist braun', der vollzieht nicht zwei Handlungen, von denen eine die bereits bekannte Sprechhandlung, dieser Tisch“, die andere ,... ist braun' ist. Die inhaltliche Fügung der Teile ist nicht das Ergebnis der aggregathaften Reihung der Handlungszüge ,dieser Tisch“ und ,... ist braun'. Daran wird zweitens sichtbar, dass Begriffe nur durch den Vollzug eines entsprechenden Urteils erworben werden können. Einen Begriff verstehen und anwenden können bedeutet, dass der Sprecher Urteile bilden und bewerten kann. Im Akt des Urteils wird uns der Sinn prädikativer Zeichen zugänglich. Demnach ist es falsch zu glauben, die beiden Teile ,Gegenstandszeichen“ und ,Begriffsausdruck' seien isoliert voneinander verfügbar und müssten lediglich zu einer Einheit verbunden werden. Das Wesen eines Prädikats weist immer schon auf den Satz hin, sodass die Einheit des Satzes im gleichzeitigen Akt von Prädikation und Urteilsbildung zu suchen ist.

Die so veranschaulichte Rede vom handlungsbezogenen Sinn der Zeichen, davon, dass ihre semantische Rolle etwas mit dem Gebrauch zu tun habe, soll nicht den Eindruck erwecken, ich würde es ablehnen zu sagen, dass Prädikate Eigenschaften in die Rede einführen oder dass in einer elementaren Aussage einem Gegenstand eine Eigenschaft zugesprochen wird. An dieser Redeweise gibt es nichts auszusetzen. Entscheidend ist primär die Reihenfolge der Erklärung. Aus pragmatischer Sicht rechtfertigt der Handlungsvollzug, den wir mit bestimmten Zeichen ausüben, die Rede vom ,Zuschreiben einer Eigenschaft'. In einem abgeleiteten Sinne kann gesagt werden, dass Eigenschaften und Begriffe ungesättigt, Gegenstände dagegen gesättigt sind - soweit klar ist, dass der Zeichengebrauch für diese inhaltliche Deutung verantwortlich ist.

Fassen wir zusammen: Meiner positiven und an Wittgenstein orientierten Lesart zufolge erklärt Frege die Einheit des Satzes unter Verweis auf vollständige und ergänzungsbedürftige Elemente, wobei die pragmatische Deutung der logischen Grundbeziehung $(F(a))$ einfachster singulärer Aussagen wie folgt aussieht: Der Handlungsvollzug der Zuschreibung einer Farbe, Form oder Größe (... ist F) ist auf einfachere sprachliche Kompetenzen angewiesen, zum Beispiel auf die Äußerung eines Namens (a). Alle konkreten Einheitsbildungen dieser Art beschreibt Frege homogen als das Fallen eines Gegenstandes unter einen Begriff. Will man diese Gleichbehandlung und Homogenität auf die pragmatische Handlungsebene übertragen, dann liegt es nahe zu sagen, dass der modifizierende Zeichengebrauch eines prädikativen Ausdrucks - egal, ob es um Farben, Größen, Formen etc. geht - stets auf gleiche Weise auf ein gesättigtes Zeichen Bezug nimmt. Bevor ich im nächsten Abschnitt auf Davidson eingehe, sei abschließend folgende Frage als Warnschild platziert: Ist es plausibel, davon auszugehen, dass die Einheit des Satzes, wenn sie im Sinne der logischen Grundbeziehung Freges verstanden wird, in allen Fällen elementarer prädikativer Vollzüge auf das Fallen eines Gegenstandes unter einen Begriff zurückgeführt werden kann? Oder anders: Muss die prädikative Handlung der Ergänzung stets nach einem inhaltlich gleichartigen Paradigma (Gegenstand und Eigenschaft) rekonstruiert werden? Frege beantwortet diese Frage ebenso affirmativ wie Davidson. An diesem Punkt setzen meine Bedenken an. 


\section{Wahrheit, Erfüllung und Prädikation: Davidsons Ansatz}

Ich wende mich nun dem Ansatz Davidsons aus Truth and Predication zu. Davidson liest Frege primär auf den Bezug der Zeichen bezogen und kommt demnach folgerichtig zu dem Schluss:

„If predicates do not refer to functions, then Frege's bold proposal is not a solution to the problem of predication." ${ }^{\text {8 }}$

Wie angedeutet, teile ich diese schwache Lesart Freges nicht. Da es mir jedoch nicht um eine Frege-Interpretation geht, will ich diesen Punkt nicht weiter verfolgen, lediglich anmerken, dass meine auf den Sinn von Zeichen und damit auf ihren Gebrauch bezogene Lesart Freges dazu führt, dass der Schritt von Frege zu Davidson (beziehungsweise Tarski) wesentlich kleiner ausfällt, als Davidson diesen beschreibt. Diese Bemerkung ist wichtig, weil die Tatsache, dass Davidson das Problem der Einheit des Satzes in meinen Augen nicht zufriedenstellend löst, gerade etwas damit zu tun hat, dass auch Freges Ansatz der logischen Grundbeziehung nicht zum Ziel führt. Aber gehen wir der Reihe nach vor.

Eine Schlüsselrolle nimmt für Davidson, wie der Titel des Buches erahnen lässt, die Beziehung zwischen Wahrheit und Prädikation ein. Eine Adäquatheitsbedingung einer jeden Theorie der Einheit des Satzes lautet:

„[...] a satisfactory account of predication depends on relating it to the truth of sentences. ${ }^{\text {"9 }}$

Dieser Forderung kann auf einer intuitiven Ebene erst einmal zugestimmt werden, haben wir die Einheit des Satzes zu Beginn doch wie folgt umschrieben: Sätze benennen nicht nur, sondern sie sagen etwas aus - das heißt, sie drücken einen wahrheitsfähigen Gedanken aus. Wo die Namensliste zu der Reihung ,Peter, Johanna, Liebe“ kommt, spricht der Satz davon, dass Peter Johanna liebt.

Nun kann es nicht das Ziel Davidsons sein, auf diese intuitive Redeweise zu verweisen. Einen theoretischen Anspruch verfolgend, stellt er darüber hinausgehend fest:

„Theories of truth and theories of predication are closely related: it seems probable that any comprehensive theory of truth will include a theory of predication. ${ }^{\text {"10 }}$

Der Bezug auf eine Wahrheitstheorie geht über die Idee hinaus, dass Sätze etwas aussagen. Gleichzeitig sollte das Einschlagen eines solchen Weges nicht überraschen, hat Davidson doch in vielen Aufsätzen für eine wahrheitskonditionale Theorie der Bedeutung argumentiert. Gehen wir also der Frage nach, was es bedeuten soll, dass eine Wahrheitstheorie im Anschluss an Tarski eine Theorie der Prädikation enthält.

Der wahrheitskonditionale Ansatz Davidsons verfolgt den systematischen Anspruch, über Bedeutungsaxiome sprachlicher Zeichen und rekursiv anwendbare Fügungsweisen die Bedeutung eines beliebigen Satzes einer Sprache L durch Bedeutungstheoreme, auszurechnen'. Diese Theoreme haben die folgende Form: ,s ist wahr genau dann, wenn $\mathrm{p}^{6}$ - wobei $\mathrm{p}$ die Beschreibung des Satzes s in einer Metasprache ist. Dieser Ansatz basiert auf der Idee, dass es eine

8 D. Davidson, Truth and Predication, a. a. O., 136.

9 Ebd., 141.

10 Ebd., 120. 
intrinsische Beziehung zwischen Wahrheit auf der einen und Bedeutung auf der anderen Seite gibt. Unter dieser Voraussetzung besteht der wesentliche Kern der Bedeutungstheorie streng genommen jedoch nicht in den Theoremen selbst, sondern in den systematischen Bildungs- und Ableitungsregeln der Bedeutungsangaben. Um die Art des Beitrags der Wortbedeutungen zu den Wahrheitsbedingungen der Sätze bestimmen zu können, verweist Davidson auf Axiome, welche sowohl die Art als auch den konkreten und spezifischen Beitrag festlegen. Auf unser Beispiel bezogen: Das Theorem T1 „, der Tisch ist braun“ ist wahr genau dann, wenn der Tisch braun ist" ergibt sich aus den Axiomen A1 „,,der Tisch“ bezieht sich auf diesen Tisch“ und A2 „,X ist braun“ wird von braunen Gegenständen erfüllt" ${ }^{“}$. Beide Axiome zusammen bestimmen die semantische Fügung des Satzes s in T1, welche wie folgt beschrieben werden kann: Der durch A1 herausgegriffene Gegenstand erfüllt die in A2 ausgedrückte Aussagefunktion. Der axiomatisch-systematische Charakter einer solchen Wahrheitssemantik verlangt, dass das Vorkommen eines Begriffes, in welchen Satzzusammenhängen auch immer, stets mit dem gleichen semantischen Beitrag verbunden ist, wobei zugestanden wird, dass es kontextuelle und auf die konkrete Äußerung bezogene Modifikationen geben kann. Metaphern beispielsweise drücken für Davidson aus, was die Wörter gemäß der Axiome und ihrer Fügung bedeuten, sodass der metaphorische Satz wörtlich gesehen falsch ist, seine Nützlichkeit dann in einem zweiten Schritt durch pragmatische Merkmale erklärt werden muss. Solche Modifikationen verändern also den gleich bleibenden Gedanken des Satzes. Nur so kann sichergestellt werden, dass die Bedeutung eines neuen Satzes aus den Teilbedeutungen und der Fügungsweise abgeleitet werden kann. Auf die Rolle des Prinzips der Kompositionalität komme ich am Ende des Aufsatzes zurück.

Für das Verständnis der Prädikation und damit für Davidsons Umgang mit dem Problem der Einheit des Satzes ist der Begriff der Erfüllung ausschlaggebend. Diesen übernimmt er von Tarski, ebenfalls dessen semantische Interpretation der Erfüllungsrelation, wonach ein Gegenstand y die Funktion ,... ist rot $^{\star}$ genau dann erfüllt, wenn y rot ist, beziehungsweise allgemeiner gesagt: F wird von a erfüllt, wenn der Satz ,a ist $\mathrm{F}^{6}$ wahr ist. ${ }^{11}$ Hier haben wir Davidsons Antwort auf die Frage nach der semantischen Komplexität vor uns. Aussagefunktionen werden durch Gegenstände beziehungsweise Sequenzen von Gegenständen erfüllt.

Auffallend an dieser Antwort ist nun erstens, dass sich diese Strategie kaum von derjenigen Freges oder Quines unterscheidet. Der Erfüllungsbegriff leistet nicht mehr - aber auch nicht weniger - als die auf den Sinn sprachlicher Zeichen bezogene Redeweise von der logischen Grundbeziehung, davon, dass ein Gegenstand unter einen Begriff fällt. Freges ungesättigte Begriffe fasst Davidson unter die Rubrik der Aussagefunktionen. In beiden Fällen läuft es darauf hinaus, dass Gegenstände ein Prädikat erfüllen beziehungsweise vervollständigen, wobei der funktionale Charakter des Prädikats bereits das Verstehen der Einheit des Satzes mit sich bringt. Gleiches lässt sich hinsichtlich der Beziehung zwischen Quine und Davidson sagen. In Word and Object bietet Quine das folgende Verständnis atomarer beziehungsweise elementarer Prädikation an: Elementarsätze werden gebildet, indem ein Gegenstand mittels singulärem Term herausgegriffen und von diesem mittels prädikativem Ausdruck etwas ausgesagt wird, wobei gilt: ,[...] predicates are true of particulars." ${ }^{\text {"12 }}$ Auch hier können wir sagen, dass Davidsons Erfüllungsrelation nicht mehr sagen soll, als das Prädikate durch Gegenstände wahr oder falsch gemacht werden.

Zusammenfassend kann festgehalten werden, dass Frege, Quine und Davidson, bei allen Unterschieden, auf eine gemeinsame Idee zusteuern, der zufolge die Einheit des Satzes

11 Die durchaus interessante Frage nach der explanatorischen Reihenfolge der Begriffe Wahrheit und Erfüllung können wir an dieser Stelle außer Acht lassen.

12 Vgl. W. V. O. Quine, Word and Object, Cambridge/Mass. 1960, 96. 
dadurch erklärt werden kann, dass erstens auf die durch die Subjektausdrücke herausgegriffenen Entitäten verwiesen wird und zweitens Begriffe mit der Aufgabe belegt werden, eine wahrheitswertfähige Einheit zu bilden. Dabei wird diese Art der semantischen Fügung für alle singulären Elementarsätze der logischen Form F(a) in Anschlag gebracht. Logisch gesehen fällt stets ein Gegenstand unter einen Begriff.

Dieser Gesichtspunkt der universalen Anwendbarkeit zeigt sich zweitens auch in der Tatsache, dass die Wahrheitssemantik Davidsons mit einen minimalen Begriff der Wahrheit auskommt. ${ }^{13}$ Das heißt: Die Bedingungen, die ein Satz erfüllen muss, um wahrheitswertfähig sein zu können (um über Wahrheitsbedingungen zu verfügen), sind einzig minimaler Art, da sie bereits durch die deklarative Oberfläche der sprachlichen Form der Sätze, verbunden mit gewissen normativen Merkmalen des Gebrauchs, als erfüllt angesehen werden können. Ähnlich äußert sich auch Wittgenstein:

,$[\ldots]$ Nun ist aber, $\mathrm{p}$ ist wahr ${ }^{6}=\mathrm{p}$ und, $\mathrm{p}$ ist falsch ${ }^{6}=$ nicht-p. Und zu sagen, ein Satz sei alles, was wahr oder falsch sein könne, kommt darauf hinaus: Einen Satz nennen wir das, worauf wir in unserer Sprache den Kalkül der Wahrheitsfunktionen anwenden." ${ }^{14}$

Daraus folgt, dass der Wahrheitsbegriff keine substanzielle Eigenschaft in die Rede einführt, wir vielmehr in der Regel dann, wenn das Äußern einer Proposition p Sinn macht, auch sagen können, dass $\mathrm{p}$ wahr oder falsch ist. Die Zuschreibung von Wahrheitsbedingungen ist demzufolge ebenso unproblematisch, damit aber eben auch sprachphilosophisch ohne explanatorischen Wert, weil eine solche Zuschreibung vom konkreten Inhalt des Sprachspiels unabhängig ist. Mit anderen Worten: Die wahrheitskonditionalen Theoreme sind auch dann für die Angabe der Satzbedeutung relevant, wenn die übergeordneten sprachphilosophischen Fragen danach, wie modale, moralische oder ästhetische Sätze funktionieren, als nach wie vor offen angesehen werden. Fragen dieser Art nehmen darauf Bezug, ob die in diesen Sprachspielen ausgeführten Züge in einem tiefer gehenden Sinne als wahrheitsfähig, wahrheitskonditional etc. beschrieben werden sollten, ob mit der minimalen Beschreibung unser Verständnis vertieft wurde oder nicht. Dabei wird nicht schon impliziert, Wahrheit sei eine metaphysische Eigenschaft, sondern im Mittelpunkt des sprachphilosophischen Interesses stehen beispielsweise Unterschiede zwischen der Beschreibung mittelgroßer Trockengüter und moralischer Werte, welche mit Bezug auf den Begriff der Wahrheit vielleicht erhellt werden können. Diese Fragen ausblendend, gibt Davidson die Bedeutung der entsprechenden Sätze durch Angabe der Wahrheitsbedingungen wie folgt an:

T1: „Der Tisch ist braun“ ist wahr genau dann, wenn der Tisch braun ist.

T2: „Der Tisch ist schön“ ist wahr genau dann, wenn der Tisch schön ist.

T3: „Die Summe von 2 und 3 ist notwendig 5“ ist wahr genau dann, wenn die Summe von 2 und 3 notwendig 5 ist.

T4: „Das Töten von Katzen ist moralisch verwerflich“ ist wahr genau dann, wenn das Töten von Katzen moralisch verwerflich ist.

13 Diese These ist alles andere als unstrittig. Davidson lehnt zwar substanzielle Wahrheitstheorien ab, glaubt aber, deshalb noch nicht auf einen Deflationismus festgelegt zu sein. Um dieser Zwischenstellung gerecht zu werden, spreche ich von einem minimalen Wahrheitsbegriff. Später werden wir sehen, dass die Frage sehr interessant und schwer zu beantworten ist, wie viel Inhalt in diesem Begriff der Wahrheit steckt (vgl. Abschnitt IV).

14 Vgl. L. Wittgenstein, Philosophische Untersuchungen, 12. Aufl., Frankfurt/M., § 136. 
Dieser Minimalismus bezüglich Wahrheit und Wahrheitsbedingungen führt uns nun zum eben erwähnten ersten Punkt zurück, da sich der Minimalismus von Wahrheit auf Erfüllung überträgt. Die semantische Fügung der Sätze in T1 bis T4 wird homogen beschrieben im Sinne des Paradigmas ,Gegenstände erfüllen Prädikate‘: Der Tisch macht die Aussagefunktion ,... ist braun' ebenso wahr wie ,... ist schön'; der Handlungstyp des Tötens von Katzen erfüllt das Prädikat ,... ist moralisch verwerflich“; und ganz analog dazu gilt, dass die Summe von 2 und 3 die Aussagefunktion ,... ist notwendig 5 $5^{\circ}$ wahr macht. Hierbei handelt es sich um eine Vereinheitlichung, die in meinen Augen nur auf der sprachlichen Oberfläche besteht, bezogen auf die inhaltliche Fügung jedoch in die Irre führt. Gegen das folgende Bild werde ich argumentieren: In allen diesen verschiedenen Sprachspielen besteht die inhaltliche Komplexität des sprachlichen Handlungsvollzuges darin, dass ein Gegenstand ein Prädikat erfüllt, sodass die vorhandenen Differenzen einzig die unterschiedlichen Gegenstandsarten (Tische, Zahlen, Modalitäten, mentale Zustände etc.) und die Vielfalt der zugeschriebenen Eigenschaften (Schönheit, Farbe, moralischer Wert etc.) betreffen.

Dass Davidson mit Frege in diesem Punkt übereinstimmt, macht folgendes Zitat deutlich:

„Sätze bedeuten das, was sie faktisch bedeuten, wegen der semantischen Eigenschaften der in ihnen enthaltenen Wörter und Verknüpfungspartikeln. Einen Satz würde man nicht verstehen, wenn man nicht wüsste, worauf sich die darin enthaltenen Namen und sonstigen singulären Termini beziehen sollen oder wenn man von der Extension der Prädikate keine Ahnung hätte. Doch wenn man darüber Bescheid weiß, weiß man auch, dass das für Wahrheit und Falschheit erforderliche Rohmaterial gegeben ist. "15

Nach Davidson verstehen wir also einen Satz, wenn die folgenden Axiomformen mit Inhalt gefüllt werden können: Der singuläre Term $\mathrm{N}$ bezieht sich auf ...; das Prädikat $\mathrm{P}$ trifft auf Dinge zu, die ... sind. Die oben erwähnten Beispiele sollten andeuten, dass diese Analyse nicht für alle Fälle zutrifft. Wer beispielsweise Sätze über Empfindungen oder moralische Zusammenhänge verstehen will, der muss in einem entscheidenden Sinne mehr leisten, als lediglich sagen zu können, welcher, Gegenstand‘ unter welchen ,Begriff‘ fällt. Darauf komme ich später zurück.

In der homogenen Behandlung der Einheit des Satzes spiegelt sich bei Davidson das Quinesche Prinzip wider, wonach, existieren' bedeutet, der Wert einer Variablen zu sein. Nach Quine sollten wir dort von Gegenständen sprechen, wo über etwas quantifiziert werden kann. Dieses Prinzip sichert die Annahme, dass singuläre Ausdrücke (unter der Bedingung, wir hätten die logische Form des Satzes offen gelegt) für Gegenstände stehen. Wo von Wahrheit die Rede ist, da muss etwas von etwas ausgesagt werden, wobei die singulären Terme auf das Bezug nehmen, was durch das zweite ,etwas' angedeutet wird. Die Leichtigkeit, von Gegenständen und Wahrheit zu sprechen, führt Davidson dann beispielsweise in seiner Adverbientheorie dazu, Ereignisse als Entitäten ins Spiel zu bringen. Adverbien werden als prädikative Zeichen aufgefasst, die von gewissen Entitäten etwas aussagen, in diesem Fall von Ereignissen. Den Fall der Adverbien verallgemeinernd, schreibt Davidson:

„Wenn man ein schlichtes Gemüt hat und globale Formen der Skepsis außer acht lässt, fällt es schwer zu akzeptieren, dass alle Sätze über Ursache und Wirkung, über Früher und Später [...] falsch sind. Sofern wir nicht völlig im Irrtum sind über die Natur der Welt [...] müssen Ereignisse zum Mobiliar der Welt gehören. " ${ }^{16}$

15 Vgl. D. Davidson, Die Rehabilitierung der Wahrheit, in: ders., Wahrheit, Sprache und Geschichte, Frankfurt/M. 2008, 44.

16 Vgl. ders., Methode und Metaphysik, in: ders., Wahrheit, Sprache und Geschichte, a. a. O., 84. 
Das heißt: Wenn die Sätze wahr sind, dann muss es etwas geben, wovon die Rede ist, in diesem Fall also von Ursachen und Wirkungen. Davidsons Ereignisontologie scheint durch die sprachliche Form der Darstellung erzwungen zu sein. Wittgenstein sieht einen verhängnisvollen Fehler darin, überall dort, wo wir auf Subjekte der Rede treffen, nach einem ,Ding Ausschau zu halten, für das das Zeichen steht. Statt Ereignisse als Entitäten einzuführen, plädiert Wittgenstein für eine andere Deutung der semantischen Komplexität dieser Sätze. Dass wir über Ursachen, Wirkungen, Handlungen und mentale Zustände reden, impliziert nicht zwingend, dass es sich hierbei um Gegenstände handelt.

Fassen wir zusammen: Die Stärke des Minimalismus in der Wahrheitssemantik und Erfüllungsrelation wird bezüglich der Frage nach der Einheit des Satzes zu einer wesentlichen Schwäche, da der konkrete Inhalt der Sätze (und damit das Sprachspiel) aus der Betrachtung herausfällt. So wie wir bezüglich der Theoreme einer Wahrheitssemantik einwenden können, dass der ganze sprachphilosophische Witz von der Objekt- auf die Metasprache verschoben wird, so gilt doch auch in gleicher Weise, dass bezüglich der Einheit des Satzes alles davon abhängt, was es konkret bedeuten soll, dass ein Gegenstand ein Prädikat erfüllt. Wie ist die Rede von mathematischen, modalen und mentalen ,Gegenständen' zu verstehen und inwieweit kommt einem Handlungstyp eine moralische Eigenschaft so zu wie dem Tisch die Farbe? Davidson bleibt uns eine Antwort schuldig. Seine These erweckt den Eindruck, mit dem Verweis auf Erfüllung sei alles gesagt, was hierzu philosophisch angeführt werden kann. Er kommt zu dem Schluss, dass jeder Versuch, mehr zu sagen, weniger ist: „What more can we demand? I think the history of the subject has demonstrated that more would be less?" ${ }^{\prime 17}$ Ich denke, dass Davidson nicht ganz Unrecht hat. Wir müssen versuchen zu verstehen, in welchem Sinne wir darüber hinausgehen können und in welchem nicht.

\section{Bilden Aussagen eine theoretische Art?}

Dass es noch einen philosophischen Spielraum jenseits der Rede von Erfüllung gibt, habe ich bereits angedeutet. Frege, Quine und Davidson vollziehen, jeweils auf ihre Weise, einen ähnlichen argumentativen Schritt der Vereinheitlichung, wenn sie, ausgehend von der einheitlichen Oberflächenform der Sätze, ein ebenso einheitliches Verständnis der semantischen Fügung postulieren. Was auf der logisch gereinigten Subjekt-Prädikat-Oberfläche (oder: Gegenstand-Begriff, Ding-Eigenschaft, Täter-Tätigkeit etc.) homogen erscheint, ist jedoch auf der Ebene des Sprachspiels mit einer Vielfalt von Handlungsformen verbunden.

Die Differenz zwischen Oberflächen- und Tiefengrammatik macht ein Dilemma deutlich, in welches sich Davidson hineinmanövriert. Entweder versteht Davidson die einheitsbildende Kraft der Prädikation minimal, oder aber er plädiert darüber hinausgehend für eine inhaltlich konstante Art der Fügung. Ist ersteres der Fall, dann hat er auf der Stufe der grammatischen Satzoberfläche zwar Recht mit der These, dass Gegenstände (der Rede) Prädikate erfüllen. Jedoch bleiben unter dieser Voraussetzung viele inhaltliche Fragen unbeantwortet. Wenn es ihm dagegen um ein substanzielleres Verständnis der Erfüllung geht, dann ist die These für einige Fälle der tiefengrammatischen Komplexbildung durchaus plausibel, für andere dagegen nicht. Auf Grund des universalen Anspruchs der Theorie läuft dieses Eingeständnis auf die Falschheit des Ansatzes hinaus.

17 Ders., Truth and Predication, a. a. O., 161. 
Davidson schwankt immer wieder von einem Horn des Dilemmas zum anderen. Das Abwechseln von formaler und inhaltlicher Lesart zeigt sich bereits am unterstellten Wahrheitsbegriff. Der Begriff, Wahrheits-Minimalismus‘ sollte anzeigen, dass es weder um eine metaphysisch robuste Theorie der Wahrheit noch um einen reinen Deflationismus geht. Davidson hat immer wieder betont, dass robuste Korrespondenz nicht zu denken ist, wissen wir doch nicht, was es bedeuten soll, sprachliche Stücke mit nicht-sprachlichen Tatsachen zu vergleichen. Daraus zieht er jedoch nicht den Schluss, dass, ist wahr' redundant, trivial ist oder in Zitattilgung aufgeht. Denn Wahrheit ist für Davidson für Bedeutung unerlässlich, sie eröffnet dem radikalen Interpreten einen Zugang zur Bedeutung über das kausale Wechselspiel von Welt, mentalen Zuständen und sprachlichen Äußerungen anderer. Ein solches Wechselspiel kausaler Art impliziert jedoch einen Begriff der Wahrheit, der über deflationistische Eigenschaften hinausgeht. ${ }^{18}$ Wenn das aber so ist, dann ist unklar, wie moralische oder religiöse Sätze in diesem Sinne wahr sein sollen.

Das Hin- und Herschwanken zwischen minimaler und inhaltlicher Deutung der Wahrheit, der Erfüllung und damit der Einheit des Satzes weist darauf hin, dass wir mit zwei ,Einheiten des Satzes', einer oberflächen- und einer tiefengrammatischen, rechnen müssen. Ich will nun damit beginnen, der Frage nachzugehen, ob es plausibel ist, eine homogene Geschichte der Einheit auf der tiefengrammatischen Ebene zu erzählen. Dazu ist hilfreich, auf ein Unverständnis Michael Dummetts bezüglich der Spätphilosophie Wittgensteins aufmerksam zu machen. Im Mittelpunkt steht dabei die Frage, welche bedeutungstheoretischen Implikationen die These „Die Bedeutung eines Wortes ist sein Gebrauch in der Sprache“ ${ }^{\text {"19 }}$ hat. Ähnlich wie Dummett argumentiert auch Davidson für eine Restriktion der Verbindung von Bedeutung und Gebrauch. Dummett leitet seine Bedenken mit der Frage ein, ob es ein ,Sprachspiel des Aussagens' gibt:

„This naturally prompts the question whether there is after all any genuine point in grouping together all those utterances which we class as assertions. This question is answered negatively by Wittgenstein." 20

Mit anderen Worten: Bildet das Aussagen eine theoretische Art? Nun ist nicht unmittelbar offensichtlich, was diese Frage mit der Einheit des Satzes zu tun haben soll. Folgende Bemerkungen dienen als Brückenschlag: Wenn wir von ,Aussagen' und ,Wahrheit' reden, dann gilt es zu erkennen, dass diese Begriffe sich auf zwei unterschiedlichen semantischen Ebenen bewegen, auf der Ebene des Sinns und auf derjenigen der Kraft. Ein Sprechakt des Behauptens unterhält eine intrinsische Beziehung zur Wahrheit, weil sich durch die Behauptung, dass p, der Sprecher auf die Wahrheit des Sachverhalts festlegt. Andererseits gibt es Bestrebungen, das Bilden wahrheitsfähiger Gedanken tiefer zu verorten, das heißt: auf die Ebene des Sinns zu bringen. Diese Beziehung wird sprachlich unter anderem dadurch angezeigt, dass der Sinn eines Satzes als propositionaler beziehungsweise deskriptiver Gehalt verstanden wird. In diesem Sinne können wir sagen: Das Fassen eines Gedankens impliziert immer schon Wahrheit, da hier ein Gegenstand unter einen Begriff fällt, was auf der zweiten Stufe die Frage sinnvoll erscheinen lässt, ob dieser deskriptive Gehalt, ob die Wahrheit des Ganzen, angenommen, erwünscht, befohlen, hinterfragt etc. wird. Entscheidend an dieser

18 Vgl. auch ders., Truth and Predication, a. a. O., hier: 121: „Ostension doesn’t establish a norm; it simply creates a disposition, given our sense organs, and so on, that is, a conditioning of sentences to aspects of the world."

19 L. Wittgenstein, Philosophische Untersuchungen, a. a. O., § 43.

20 Vgl. M. Dummett, Frege. Philosophy of Language, 2. Aufl., Cambridge/Mass. 1981, 356. 
von Dummett und Davidson vertretenen Trennung der Ebenen Sinn und Kraft ist die Annahme, dass es eine einheitsbildende Struktur auf der vor-illokutiven (gebrauchsneutralen) Ebene des Sinns gibt, für die Davidsons These der Erfüllung eines Prädikats durch einen Gegenstand relevant ist.

Die Ausnahmestellung deklarativer Sätze wird vor diesem Hintergrund also nicht primär damit begründet, dass alle anderen illokutiven Züge im Sprachspiel inferentiell an entsprechende deklarative Sätze gebunden sind. Diese These könnte auch Wittgenstein teilen. Nichts spricht dagegen, dass das Verstehen einer Frage das Verstehen möglicher Antworten voraussetzt, der Vollzug eines Befehls semantisch an jene Sätze gebunden ist, die mitteilen, wann ein solcher erfüllt wurde, eine Wunschäußerung intrinsisch an ihre Erfüllungsbedingungen gebunden ist etc. Abgesehen von Gedankenexperimenten über andere mögliche Sprachen, gilt es festzustellen, dass unsere Sprache deklarativen Sätzen eine Schlüsselrolle zuweist. Dummett jedoch hatte einen anderen Punkt im Auge. Aus der Tatsache, dass deklarative Sätze gegenüber nicht-deklarativen Sätzen semantische Priorität besitzen, folgt nicht, dass der propositionale Gehalt deklarativer Sätze einheitlich konstruiert werden muss. Ich will damit Folgendes sagen: Ich sehe keine sprachphilosophischen Schwierigkeiten darin, ein allgemeines Sprachspiel des Aussagens inhaltlich zu kennzeichnen, wenn wir auf den Begriff der Wahrheit verzichten beziehungsweise diesen deflationistisch deuten. Pragmatische Ansätze nach Brandom oder Price ${ }^{21}$ stellen folgende Gesichtspunkte in den Mittelpunkt der Assertion: Propositionale Aussageformen platzieren sprachliche Vollzüge in den Raum der Gründe, geben die Möglichkeit, Gedanken zur Diskussion zu stellen, verlangen eine Rechtfertigung etc. In diesem Sinne können wir ,Aussagen“ als eine theoretische Art herausgreifen, da alle sprachlichen Vollzüge gewisse pragmatische Merkmale teilen. Doch damit ist erstens wenig gesagt, weil die Gebrauchsmerkmale sehr abstrakt beschrieben wurden, sodass wir nach wie vor auf das Sprachspiel verwiesen sind, wenn es darum geht zu erkennen, wie die Rechtfertigung einer moralischen oder modalen Aussage funktioniert. Daraus folgt zweitens, dass das Problem der Einheit des Satzes durch solche pragmatischen Bestimmungen nicht angegangen wird.

Woran Wittgenstein denkt und was er Dummett zufolge negativ beantwortet, ist vielmehr die Frage, ob alle Sätze der deklarativen Oberfläche auf gleiche Weise zu ihrem propositionalen Gehalt kommen, ob alles Aussagen in einer Deklaration besteht. Mit anderen Worten: Besteht auch auf der Ebene des Sinns eine theoretische Art namens ,Aussage', die der logischen Grundbeziehung Freges oder Davidsons gehorcht? Können wir sagen, dass Wahrheit immer schon im Spiel ist, sobald es um die Individuation propositionaler Gehalte geht, dass die Ebene des Sinns durch die Beschreibung eines Sachverhalts hinreichend bestimmt wurde? Dummett stimmt hierin mit Davidson überein, auch wenn seiner Meinung nach nicht Wahrheit der Schlüssel zur semantischen Komplexität ist. Dieser Punkt ist wichtig, folgt daraus doch, dass der Begriff der Wahrheit streng genommen nebensächlich ist, weil Wittgenstein in gleicher Weise jeden anderen Versuch ablehnen würde, mittels Bezug auf einen semantischen Schlüsselbegriff eine homogene Erklärung des propositionalen Gehalts zu geben. Im Herzen der Auseinandersetzung steht das Bestreben nach einer systematischen Theorie der Bedeutung:

21 R. B. Brandom, Making it Explicit, Cambridge/Mass. 1994, Kap. 3; H. Price, Immodesty without Mirrors - Making Sense of Wittgenstein's linguistic Pluralism, in: M. Kölbel u. B. Weiss (Hg.), Wittgenstein's Lasting Significance, London 2004, 179-205. 
„[...] the implicit assumption underlying the idea that there is some one key concept in terms of which we can give a general characterization of the meaning of a sentence is that there must be some uniform pattern of the derivation of all the other features of the use of an arbitrary sentence, given its meaning as characterized in terms of the key concept. ${ }^{\text {"22 }}$

Gegen Davidson und Dummett glaubt Wittgenstein, dass der Slogan ,Bedeutung ist Gebrauch ‘ bis auf den Sinn, bis auf den Begriff der Proposition und damit bis zur Einheit des Satzes selbst durchschlägt. Die Vielfalt des Gebrauchs soll die umfassende Erklärungskraft des semantischen Schlüsselbegriffs unterlaufen.

\section{Wittgensteins pragmatische Revolutionen}

Wittgensteins Diskussion semantischer Aspekte als konstituiert durch die Art und Weise des Gebrauchs sprachlicher Zeichen kann als pragmatische Revolution bezeichnet werden. Der letzte Abschnitt hat deutlich gemacht, dass die Gebrauchsthese stärker und schwächer gelesen werden kann. Das heißt: Wir müssen zwischen zwei Revolutionen dieser Art unterscheiden. Dass beide nicht immer klar voneinander getrennt werden beziehungsweise die zweite häufig gar nicht zur Kenntnis genommen wird, liegt nicht zuletzt daran, dass die relevanten Textstellen Wittgensteins unterschiedlich stark gelesen werden können. Schauen wir exemplarisch auf folgende Bemerkungen:

„Wieviele Arten der Sätze gibt es aber? Etwa Behauptungen, Frage und Befehl? - Es gibt unzählige solcher Arten: unzählige verschiedene Arten der Verwendung alles dessen, was wir ,Zeichen', ,Worte‘ und ,Sätze' nennen. [...] Es ist interessant, die Mannigfaltigkeit der Werkzeuge der Sprache und ihrer Verwendungsweisen, die Mannigfaltigkeit der Wortund Satzarten, mit dem zu vergleichen, was Logiker über den Bau der Sprache gesagt haben." 23

„Als ob es nur Eines gäbe, was heißt: ,von Dingen reden“. Während wir doch das Verschiedenartigste mit unseren Sätzen tun. "24

„Das Paradox verschwindet nur dann, wenn wir radikal mit der Idee brechen, die Sprache funktioniere immer auf eine Weise, diene immer dem gleichen Zweck: Gedanken zu übertragen - seien diese nun Gedanken über Häuser, Schmerzen, Gut und Böse, oder was immer." ${ }^{25}$

Diese Passagen legen nahe, die pragmatische Revolution zuerst darin zu suchen, dass wir von der Fokussierung auf, eine Behauptung aufstellen' befreit werden. Wer ,Feuer' ruft oder jemanden mit einer Aussage beleidigt, der beschreibt keinen Zustand der Welt. Wahrheit ist nicht immer das wesentliche Handlungsmotiv sprachlicher Vollzüge. Es gibt neben Behauptungen unzählige weitere Aspekte des Gebrauchs eines Satzes. Auf dieser Ebene der Deutung bezieht sich der Gebrauch entweder auf illokutive Rollen oder auf den Witz einer konkreten Äußerung.

22 M. Dummett, Frege, a. a. O., 362.

23 Vgl. L. Wittgenstein, Philosophische Untersuchungen, a. a. O., § 23.

24 Ebd., § 27.

25 Ebd., § 304 . 
Gleichzeitig kommt in den Textstellen jedoch auch eine stärkere Lesart zum Ausdruck, die sichtbar wird, wenn wir uns auf den Bau der Sätze konzentrieren und diesen mit der Phrase , von Dingen reden“ in Beziehung setzen. Diese Lesart greift an, was Wittgenstein an anderer Stelle das ,Satzradikal' genannt hat, eine Konstante, die in allen möglichen Verwendungen eines Satzes vorkommt, nur jeweils anders in den Handlungskontext eingebunden wird. In diesem Sinne habe ich oben von der Deklaration gesprochen. Es legt sich die Idee nahe, das Satzradikal sei wie ein Bild, das uns den beschriebenen Sachverhalt, den deskriptiven Gehalt anzeigt, den es zu verstehen gilt. ${ }^{26}$ Die von den Logikern unterschlagende Mannigfaltigkeit der Verwendung betrifft dann nicht die Kraft oder den Witz, sondern unmittelbar den Gedanken. Was Wittgenstein in diesem Zusammenhang angreift, ist nicht, dass wir unsere Züge im Sprachspiel zumeist unter die Kategorie des ,einen Gedanken mitteilen[s]' bringen, sondern primär die Vorstellung, es gäbe nur einen Sinn dessen, was es bedeuten kann, von Dingen zu reden. Die pragmatische Revolution setzt an ihrer tiefsten Stelle nicht darauf, neben Wahrheit weitere Zielpunkte des Sprechens und Denkens an Deck zu holen. Vielmehr geht es darum, selbst die auf Wahrheit bezogenen Akte ihrer Homogenität zu berauben. Natürlich können wir sagen, jeder Aussagesatz beschreibe einen Weltausschnitt, sei dieser nun ein moralischer, mathematischer, ästhetischer oder religiöser Ausschnitt. Wir sollten uns aber im Klaren darüber sein, dass mit dieser Verallgemeinerung noch nicht erläutert wurde, was es heißt, einen religiösen oder mathematischen Sachverhalt zu beschreiben. Eine Antwort auf diese Frage gibt einzig das entsprechende Sprachspiel, welches es zu durchschauen gilt, will man ein kompetenter Mitspieler des Sprachspiels sein.

An dieser Stelle deutet sich an, dass die von Davidson und Dummett vertretene Auffassung bezüglich der Trennung von Sinn und Kraft nicht aufrechterhalten werden kann. Davidson äußert sich wiederholt unentschlossen, was die Relevanz des Gebrauchs für die Bedeutung anbelangt. Diese Unentschlossenheit bringt zum Ausdruck, dass er an der Idee des gebrauchsneutralen, deskriptiven Satzradikals festhält:

„Meaning depends on use, but it is not easy to say how, for uses to which we may put the utterance of a sentence are endless while its meaning remains fixed." ${ }^{27}$

Hinter dieser These der Trennung von Bedeutung und Gebrauch steht die Idee, wir könnten in einem ersten Schritt immer angeben, worüber wir sprechen, was die Wahrheitsbedingungen sind und welchen Gedanken wir ausdrücken. Offen bleibt lediglich die konkrete Verwendung. Worauf Wittgenstein erwidert, dass in vielen Fällen gar nicht klar ist, was es bedeuten soll, von einem ,Gedanken` zu sprechen, wenn wir nicht wissen, welche Stellung im Sprachspiel der jeweilige Satz einnimmt. Er deutet zur Veranschaulichung auf folgendes Beispiel. Gegner: Angenommen, jemand sagt uns, dass Uhren die Aufgabe haben, die Zeit anzuzeigen. Damit ist gesagt, welche Information Uhren übermitteln - auch und gerade dann, wenn völlig unklar und offen ist, was die Zeit ist und wozu es überhaupt eine Praxis des Zeitangebens und -messens gibt. Wittgenstein: Aber was soll es in diesem Zusammenhang bedeuten, dass Uhren die Zeit angeben, wenn wir nicht wissen, wie die Praxis funktioniert, wie ,Zeiten ' in unserer Lebensform integriert sind etc.? An dieser Stelle verschwindet der Gedanke des Satzes. Auf die Sprache übertragen, bedeutet das: Wir befinden uns im Irrtum, wenn wir glauben, Sätzen haben einen bestimmten deskriptiven Gehalt, sagen etwas über Empfindungen, moralische Werte etc. aus, wobei die Verwendung dieser Sätze und damit ihr Sitz in unserer Lebenswelt

26 Vgl. ebd., § 249.

27 Vgl. D. Davidson, Truth and Predication, a. a. O., 123. 
erst nachträglich zu untersuchen ist, quasi von sekundärer Bedeutung draufgesattelt wird. Wittgensteins Rede vom ,Gebrauch', bezogen auf die Stellung einer sprachlichen Handlung in einem größeren (sprachlichen und nicht-sprachlichen) Handlungszusammenhang, soll nicht zuletzt sagen, dass der ,propositionale Gehalt' ${ }^{6}$ ich aus diesem allererst ergibt. ${ }^{28}$

Die Spätphilosophie Wittgensteins ist voll von Beispielen, wie die Sicht des Logikers, es gäbe nur eine Form des Satzbaus (ein Gegenstand fällt unter einen Begriff), der Grund für begriffliche Verwirrungen ist. Wer glaubt, in Aussagen über Handlungsmotive und Empfindungen wird ein ,Gegenstand' durch einen Begriff näher bestimmt, nur sei der Gegenstand eben von besonderer Art, innerlich, nicht ausgedehnt im Raum, privat etc., der versteht den Zug im Sprachspiel falsch, wird er doch durch dieses Bild zu falschen Anschlusshandlungen und -fragen verleitet: Wo ist dieser Gegenstand, wie kann man ihn am besten untersuchen, ist er mit anderen inneren Zuständen und Gegenständen identisch? Die These lautet dagegen gerade, dass in Sätzen über Zahlen, Zeiten und mentalen Zuständen jeweils andere Weisen der semantischen Komplexbildung aktiv sind, die auf der Ebene der sprachspielbezogenen Bedeutungseinheit der entsprechenden komplexen Handlung sichtbar werden. Es kommt also zu einer Diskrepanz zwischen sprachlicher Form der Darstellung und pragmatischer Einheit auf der Handlungsseite.

\section{Gedanken zur pragmatischen Genealogie sprachlicher Formen}

Wie kommt es zu dieser Diskrepanz? Beantworten möchte ich diese zentrale Frage dadurch, dass ich eine These von Hans Julius Schneider aufgreife, die er an verschiedenen Stellen durch den Begriff der , syntaktischen Metapher' zum Ausdruck gebracht hat. ${ }^{29}$ Die These lautet: Es wird davon ausgegangen, dass wir sprachliche Darstellungsformen von einem ursprünglichen Handlungskontext, in welchem die grammatische beziehungsweise logische Klassifikation der semantischen Rollen, beispielsweise von ,Gegenstand' und ,Begriff', in einem klaren Sinne inhaltlich motiviert ist, auf einen neuen Handlungsbereich übertragen, für den jedoch gilt, dass von ,Gegenständen“ im eigentlichen Sinne keine Rede ist und/ oder in dem die Modifikation beziehungsweise Sättigung des Gegenstandszeichens durch ein ungesättigtes Zeichen anders zu verstehen ist als im Ausgangsfall. Angenommen, wir verstehen im Zuge des Spracherwerbs die propositionale Sprachform in situativen Szenen, die primär damit befasst sind, Gegenstände des Handelns zu klassifizieren. Zuerst schreiben wir Dingen Eigenschaften zu, an denen wir uns stoßen, die wir von a nach b tragen, die wir verstecken, derentwegen wir uns streiten und vieles mehr. Wenn das Kind vor diesem Hintergrund lernt, dass der Ball rund ist oder Mama rennt, dann deutet die Verschmelzung der semantischen Teile auf der sprachlichen Ebene unmittelbar auf entsprechende Merkmale der Sprechhandlung hin. Die Unterscheidung von Subjekt und Prädikat impliziert auf dieser Ebene, dass ein Gegenstand eine Eigenschaft hat beziehungsweise ein Täter etwas tut. Sehr schnell kommt es dann aber zu, grammatischen Bewegungen'. Die Übertragung der an konkreten Beispielen verständlichen grammatischen Form lässt allererst eine Form im strengen

28 Ganz ähnlich argumentiert Huw Price (Immodesty Without Mirrors - Making Sense of Wittgenstein's Linguistic Pluralism, a. a. O., 185): „I want to give a pragmatic account of how there come to be descriptive contents or thoughts of particular kind.“

29 Vgl. H. J. Schneider, Phantasie und Kalkül, Frankfurt/M. 1999; ders., ,Syntaktische Metaphern“ und ihre begrenzende Rolle für eine systematische Bedeutungstheorie, in: Deutsche Zeitschrift für Philosophie, 41 (1993), 477-486. 
Sinne des Wortes entstehen. Denn die metaphorische Erweiterung der Fügungsweise führt zu einer Diskrepanz zwischen Form und Inhalt, da in Sätzen wie ,Der Ball ist schön“, ,Mama weint' oder ,Das Schreien hörte auf' entweder die Modifikation des gesättigten Zeichens ,Ball` beziehungsweise ,Mama' anders funktioniert, was sehr gut daran sichtbar ist, dass Weinen keine Tätigkeit wie Rennen ist beziehungsweise die Zuschreibung ästhetischer Attribute anderen normativen Diskursregeln gehorcht als beispielsweise die Zuschreibung einer Form. Im letzten Satz haben wir es darüber hinaus mit einer doppelten Übertragung zu tun, weil ,das Schreien' kein Gegenstand ist und ,aufhören' keine Eigenschaft oder Tätigkeit symbolisiert. Übertragungen dieser Art nehmen ihren Lauf, wenn wir lernen über Zahlen, Gefühle und andere Aspekte des Lebens zu sprechen. Wir bilden Sätze wie ,Die Zahl 5 ist ungerade“, ,Das Schöne variiert mit der Perspektive des Betrachters', ,Die Liebe ist ein komisches Ding' etc. Der Sprecher muss erkennen, dass die sprachliche Form einen Gegenstand erzwingt, damit aber nicht gesagt ist, dass Zahlen, das Schöne und die Liebe auf die gleiche Weise ,Gegenstände des unmittelbaren Umgangs' sind wie Bälle, Tische und Menschen.

Metaphorische Projektionen und ,grammatische Bewegungen' dieser Art sind uns im Alltag bestens vertraut. Ohne größere Schwierigkeiten verstehen wir Äußerungen wie ,Es schneit' oder ,Schönheit existiert'. In diesen Fällen erkennen wir, dass die grammatische Form ein Bild der inhaltlichen Fügung transportiert, welche auf der Handlungsebene jedoch nicht eingelöst wird.

Aus philosophischer Perspektive entscheidend ist nun, dass die auf diese Weise genealogisch und pragmatisch auseinanderlaufende Einheit der grammatischen und handlungsbasierten Struktur ein allgegenwärtiges Phänomen menschlicher Sprache ist, welches wir nicht immer so leicht durchschauen wie im Falle der eben erwähnten einfachen Beispiele. Wittgenstein sieht darin den Ursprung philosophischer Verwirrungen. Er schreibt:

„Dasselbe geschieht nun, wenn wir die Wirklichkeit nach der Subjekt-Prädikat Norm in unserer Sprache abbilden. Das Subjekt-Prädikat Schema dient als Projektion unzähliger verschiedener logischer Formen. “30

„Dass wir Subjekt-Prädikatsätze gebrauchen, ist nur eine Angelegenheit unserer Zeichengebung. “31

„Das heißt - so scheint es - dass der Wortlaut das Sprachspiel nicht bestimmt, in welchem der Satz funktioniert. Die logische Notation verschluckt die Struktur.“32

Gerade die letzte Bemerkung macht deutlich, dass es Wittgenstein nicht nur um die grammatischen Strukturen von Subjekt und Prädikat geht, sondern dass er Gleiches bezüglich der logischen Dimensionen ,Gegenstand“ und ,Begriff' beziehungsweise ,Aussagefunktion“ sagen würde. In allen diesen Fällen sieht er eine grammatische Fügung am Werk, die die Vielfalt der semantischen Einheitsbildung maskiert. Letztere zeigt sich im Sprachspiel.

30 Vgl. L. Wittgenstein, Philosophische Grammatik, 4. Aufl., Frankfurt/M. 1999, 205.

31 Vgl. ders., Philosophische Bemerkungen, 5. Aufl., Frankfurt/M. 1999, 119.

32 Vgl. ders., Bemerkungen über die Grundlagen der Mathematik, 6. Aufl., Frankfurt/M. 1999, 284. 


\section{Das Zusammenspiel von grammatischer und handlungsbasierter Einheit des Satzes}

Ich möchte die in den letzten Abschnitten besprochenen Punkte nun zusammenfügen. Hans Julius Schneider spricht auf Grund der Existenz syntaktischer Metaphern von zwei Ebenen des Sinns und Verstehens, einer grammatischen und einer pragmatischen. Diese Differenz können wir auf die Einheit des Satzes übertragen und dadurch wichtige Einsichten Davidsons mit den bereits angesprochenen Schwächen in ein gemeinsames Bild integrieren. In diesem Zusammenhang taucht erneut die Frage Davidsons auf, ,what more can we demand?“, die am Ende beantwortet werden soll.

Beginnen wir mit der Einheit des Satzes auf der Ebene der Sprechhandlung. Der letzte Abschnitt hat dafür argumentiert, dass, um zu wissen, welchen Gedanke ein Satz ausdrückt, der Sprecher in der Lage sein muss, die Stellung des sprachlichen Zuges im Sprachspiel zu beherrschen. Dass dieser Zug nicht in allen Fällen von der Art der logischen Grundbeziehung ist, hat Wittgenstein wiederholt deutlich gemacht. Bezogen auf die Philosophie der Psychologie heißt es:

„Man kann sich beim Sprechen auf einen Gegenstand beziehen, indem man auf ihn zeigt. Das Zeigen ist hier ein Teil des Sprachspiels. Und nun kommt es vor, als spreche man von einer Empfindung dadurch, daß man seine Aufmerksamkeit beim Sprechen auf sie richtet. Aber wo ist die Analogie? [...] Aber auch auf den Gegenstand zeigen, von dem man spricht, kann ja für das Sprachspiel, für den Gedanken, unter Umständen ganz unwesentlich sein. ${ }^{\text {"33 }}$

Der Witz mentaler Zuschreibungen, so können wir für viele Fälle sagen, hat gerade nichts damit zu tun, dass ein innerer Gegenstand postuliert wird. Schauen wir auf das Sprachspiel als das Primäre, als den Ort, an dem die Sprechhandlung arbeitet, eine Bedeutungseinheit bildet, dann fällt in diesen Fällen Folgendes auf: Wer über Vorstellungen, Motive, Gründe und Empfindungen spricht, der behauptet nicht, es gehe ihm um ein inneres Etwas, sei es nun mentaler oder physiologischer Natur, dessen genaue Identifikation noch ungeklärt ist. Die Fragen, wie man diesen inneren Gegenstand oder Prozess wahrnimmt, wo er ist beziehungsweise vor sich geht, machen in den meisten Fällen keinen Sinn, weder in der Ersten noch in der Dritten Person-Perspektive. Aus der Ersten Person-Perspektive schließen wir nicht auf Grund einer besonderen Beobachtung eines ,Dings', dass eine Empfindung oder eine Absicht vorliegt. Der eher expressive beziehungsweise konstruktive Charakter weist in die dialogische Zukunft, weniger beschreibend in die Vergangenheit. Das sieht man daran, dass die Angabe eines Grundes im fortschreitenden Diskurs nicht dadurch korrigiert wird, dass der Sprecher einen besseren Blick auf einen verborgenen Gegenstand erwirbt, sondern vielmehr wirkt die veränderte Perspektive auf sich selbst als Akteur. Ein neuer Blick modifiziert die eigene Geschichte. Wird in diesen Situationen völlig zu Recht von Wahrnehmung und Beobachtung gesprochen, dann beziehen wir uns mit dieser Rede auf Personen und Szenen. Ähnliches gilt für die Dritte Person-Perspektive. Was ich wahrnehme, ist nicht ein ,Etwas', sondern eine sich im öffentlichen Handlungsraum bewegende Person. Ein ,Etwas' taucht im Sprachspiel der Wünsche, Überzeugungen und Empfindungen nicht auf, wenngleich die sprachliche Form ein solches nahe legt. ${ }^{34}$

33 Vgl. ders., Philosophische Untersuchungen, a. a. O., § 669.

34 Vgl. dazu ebd., § 293: „Das heißt: Wenn man die Grammatik des Ausdrucks der Empfindung nach dem Muster ,Gegenstand und Bezeichnung“ konstruiert, dann fällt der Gegenstand als irrelevant aus der Betrachtung heraus." 
Ich habe angedeutet, dass die genealogische Geschichte der Übertragung grammatischer Formen auch und gerade die prädikative Erweiterung eines gesättigten Zeichens betrifft. Darauf bezogen erscheint es mir plausibel, Weisen der Prädikation zu unterscheiden. Etwas konkreter gesagt: Die Kopula funktioniert auch dann nicht homogen, wenn es um die Zuschreibung einer Eigenschaft geht. Das habe ich an einigen Beispielen bereits angedeutet. Exemplarisch sei hier auf die beiden Sätze verwiesen ,Der Hund Struppi ist vierbeinig‘ und ,Der Hund Struppi ist verschwunden'. Die auf die Oberfläche der Sätze bezogene Sprechhandlung der Prädikation vereint inhaltlich sehr unterschiedliche Fälle. Wir würden nicht sagen wollen, dass dem Hund die Eigenschaft des Verschwundenseins auf ähnliche Weise zukommt wie die der Vierbeinigkeit.

Ein anderer Fall stammt aus der Metaethik. An dieser Stelle sei explizit etwas zur Funktion moralischer Sätze angeführt. Gegen Davidson halte ich daran fest, dass es gute Gründe gibt, diese auf der Ebene des Sprachspiels nicht im Sinne des Wahrheits-Paradigmas zu rekonstruieren. Deshalb habe ich meine Zweifel, Handlungen als ,Ereignisse' zu klassifizieren, denen dann moralische oder empirisch-deskriptive Eigenschaften zugesprochen werden. Ein solcher Zug vernachlässigt die Logik der Differenzen. Wer sagt, dass das Töten von Katzen moralisch verwerflich ist, der beschreibt kein Ereignis, sondern bringt eine normativ geladene, handlungsanleitende Einstellung zum Ausdruck. Als natürliche Wesen bilden wir in Konfrontation mit der Welt Einstellungen unterschiedlichster Art aus, von denen einige als ,moralisch ' angesehen werden. Wollen wir das Sprachspiel moralischer Urteile verstehen, dann müssen wir die Geschichte der rationalen Einstellungsausbildung und ihre Stellung in unserer Handlungswelt verstehen. Auch wer diesen an Hume angelehnten Zugang zur Moral nicht teilt, sollte mir zustimmen können, dass Handlungen auf eine andere Weise moralische Eigenschaften haben, als ihnen in der Regel körperliche Merkmale zukommen. Ich sehe bezogen auf den Vergleich zwischen empirisch-deskriptiven, moralischen, modalen und religiösen Urteilen nicht verschiedene Instantiierungen einer Komplexbildungsweise, sondern verschiedenartige Modifikationen.

Gehen wir nun zur grammatischen (beziehungsweise logischen) Rede von der Einheit des Satzes über. Wittgenstein behandelt die Fregesche Unterscheidung von Gegenstand und Begriff - und damit indirekt auch den Ansatz Davidsons - als an der sprachlichen Oberfläche orientiert. Die Differenzierung zwischen zwei Einheiten des Satzes gibt ihm darin teilweise Recht. Dennoch dürfen wir nicht übersehen, dass Frege und Davidson in einem wichtigen Punkt Recht haben. Die Klassifikation der semantischen Teile im Satz als Gegenstandsname und prädikatives Zeichen sagt etwas über die semantische Rolle der Zeichen im Satzzusammenhang. Das Auffassen der logischen Kategorien ist ein wichtiger Bestandteil des Verstehens der Satzbedeutung. Diese Rollen hat Frege unter die Begriffe ,gesättigt' und ,ungesättigt' gebracht. Auf den Gebrauch bezogen und vor dem Hintergrund der genealogischen Ausbuchstabierung der grammatischen Form steht die grammatische Klassifikation immer auch für eine inhaltliche Beziehung der Satzteile. Wer in dem Satz ,der Tisch ist braun ${ }^{6}$ zwischen Gegenstandsname und Begriff unterscheiden kann, der besitzt damit auch ein Wissen darüber, wie die Teile in anderen Handlungskontexten funktionieren, das heißt in welchen Zusammenhängen wir von Tischen sprechen und wie die Zuschreibung einer Farbeigenschaft funktioniert. Durch diese Verknüpfung von grammatischer Klassifikation und inhaltlicher Verbindung wird ein holistisches Netz begrifflicher Beziehungen geknüpft. Auf dieser Ebene ist es dann in vielen Fällen möglich, die Bedeutung eines neuen Satzes aus der Bedeutung der Teile und der bereits bekannten Fügungsweise abzuleiten. Wenn wir das Bauarbeiter-Sprachspiel Wittgensteins zu Beginn der Philosophischen Untersuchungen so weitererzählen, dass die beteiligten Personen neben den Äußerungen ,Platte!‘, ,Würfel!` und ,Säule!‘ auch den 
komplexen Satz, Die Platte ist zerbrochen“ beherrschen, dann können wir uns leicht ausmalen, dass das Verstehen des neuen Satzes ,Der Würfel ist zerbrochen' auf Anhieb verstanden wird. Das Verstehen der grammatischen Einheit des Satzes ergibt sich in diesem Sinne aus den Teilen, und es führt in vielen Fällen zum Gedanken des Satzes - in vielen, nicht in allen Fällen. Frege und Davidson betonen diese Ebene also völlig zu Recht. Unsere grammatischen Kompetenzen als Sprecher einer Sprache können nicht vernachlässigt werden, weil sie erstens Aufschluss über die Worten im Satz geben und zweitens, daran anknüpfend, immer auch auf Handlungskontexte verweisen, in denen die Zeichen und ihre Fügung auf eine inhaltliche Weise bereits verstanden werden. Der Sinn logischer Kategorien spiegelt Handlungsmöglichkeiten der Lebensform wider.

\section{Einige Konsequenzen}

Statt einer Zusammenfassung möchte ich einen Ausblick auf einige Konsequenzen des vorgestellten Ansatzes geben. Erstens: Das leitende Motiv des Strebens nach einheitlicher Komplexbildung ist die Konstruktion einer systematisch-axiomatischen Theorie der Bedeutung. Davidsons Überzeugung, dass , ,...] it is important that truth $[\ldots]$ is a unitary property, for it is this that ties it so closely to the problem of predication“"35, macht nur vor der Annahme Sinn, es ließe sich für jeden Satz einer Sprache mittels Axiomen und rekursiven Fügungen angeben, welcher Gedanke zum Ausdruck gebracht wird. Hans Julius Schneider hat dagegen die begrenzende Rolle der syntaktischen Metapher für ein solches Projekt betont. ${ }^{36}$ Die Übertragung der Fügungsweise macht ein auf den Einzelfall bezogenes Eintauchen ins Sprachspiel unerlässlich. Insoweit ist Wittgenstein mit seiner, Systemkritik' im Recht.

Zweitens: Das heißt nun aber auch, dass das Prinzip der Kompositionalität nicht allgemein gültig sein kann. Die Bedeutung eines noch nicht bekannten Satzes kann nur dann aus den semantischen Teilen und der Art ihrer Zusammensetzung abgeleitet werden, wenn keine metaphorische Projektion vorliegt, wenn also die Teile und die Komplexbildungsweise aus anderen Kontexten bereits bekannt sind. Dagegen gibt es Fälle, in denen dieses Wissen nicht ausreicht, um den ausgedrückten Gedanken zu erfassen. Wittgenstein erwähnt in unterschiedlichen Zusammenhängen beispielsweise ,Ich bin hier' und ,Eine Rose hat keine Zähne‘. Obwohl wir mit den Teilen vertraut sind und sicher auch Beispiele für die Fügungsweise angeben können, ist alles andere als klar, was diese ,Sätze' bedeuten sollen. Die Unklarheit ist ein Unverständnis darüber, wie ein entsprechendes Sprachspiel aussehen könnte.

Drittens: Kommen wir auf Davidsons Frage und seine Antwort zurück: „What more can we demand? I think the history of the subject has demonstrated that more would be less?" Meines Erachtens sollten wir auf diese Frage mit einem Ja und Nein reagieren. Wer in systematischer Hinsicht etwas universal Gültiges über die Einheit des Satzes sagen will, dem bleibt letztendlich aus guten Gründen die logische Klassifikation von ,Gegenstand ' und ,Aussagefunktion“ übrig. Im letzten Abschnitt wurde festgehalten, dass das Erkennen der grammatischen Form in vielen Fällen zum Verstehen des Handlungssinns führt. Selbst wenn das nicht eintritt, so bleibt das Auffassen dieser Kategorien doch eine Voraussetzung für das Verstehen des metaphorischen Schrittes. In diesem Sinne hat Davidson Recht. Wittgensteins

35 Vgl. D. Davidson, Truth and Predication, a. a. O., 130.

36 Vgl. H. J. Schneider, Syntactic Metaphor: Frege, Wittgenstein, and the Limits of a Theory of Meaning, in: Philosophical Investigations, 13 (1990), 137-153. 
Methode des Vorführens und Durchsichtigmachens konkreter Sprachspiele zeigt aber auch, dass es hier bei weitem mehr zu sagen gibt, als Davidson sieht.

Viertens: Abschließend sei eine Konsequenz erwähnt, die ein eigenes Vorhaben meinerseits betrifft. An einigen Stellen des Textes habe ich auf non-faktualistische Argumente aufmerksam gemacht. Non-Faktualistische Semantiken richten sich gegen die These, die Sprache funktioniere (fast) immer beschreibend. In meinen Beispielen habe ich auf moralische, modale und religiöse Urteilspraktiken verwiesen, in denen mir diese These auf nahe liegende Weise falsch zu sein scheint. Die Rede von moralischen, modalen und religiösen Tatsachen ist das, was erklärt werden muss und deshalb im Zuge einer sprachphilosophischen Geschichte nicht vorausgesetzt werden kann. Mit Simon Blackburn gesprochen: Diese Tatsachen und Gegenstände sind die Kinder, nicht die Eltern des Sprachspiels. Dennoch stehen non-faktualistische Ansätze gegenwärtig auf schwachen Füßen. Das hängt nicht zuletzt damit zusammen, dass sie im Sinne der Expression eines inneren Zustandes häufig psychologistisch auf den Weg gebracht werden. Wenn Sprecher mit ihren Äußerungen nicht beschreiben wollen, sondern vielmehr eine innere Einstellung nach außen bringen, dann sollten Non-Faktualisten zwei Fragen beantworten können: Von welcher Art ist der mentale Zustand, und wie ist der Vorgang der Expression zu verstehen? Ich plädiere dagegen für eine semantische Fundierung des Non-Faktualismus, welche den eigentlichen Witz dieser Ansätze mit dem Problem der Einheit des Satzes in Verbindung bringt.*

Stefan Tolksdorf, Technische Universität Berlin, Institut für Philosophie, Wissenschaftstheorie, Wissenschafts- und Technikgeschichte, Straße des 17. Juni 135, 10623 Berlin

\begin{abstract}
The paper explores insights and limits of Davidson's minimalistic approach to the problem of the unity of proposition. It argues that the minimalistic way to explain the unity, namely that an object satisfy a predicate, remains at the surface grammar. The author tries to show how Wittgenstein's later philosophy of language opens up a second level of unity, i. e. a unity of the speech action (depth grammar). It follows that we have to differentiate two kinds of unity-problems, so that Davidson only offers an answer to the first one. The paper ends by drawing some ramifications for the principle of compositionality, theories of meaning and semantic non-factualism.
\end{abstract}

* Teile dieses Aufsatzes wurden auf einem Doktorandenworkshop der Gesellschaft für analytische Philosophie (GAP) 2008 in Regensburg vorgetragen. Ich danke den Veranstaltern und Referenten für viele hilfereiche Bemerkungen. Darüber hinaus möchte ich Christoph Caspar Pfisterer und Hans Julius Schneider für Besprechungen des Manuskripts danken. 